\title{
Research Paper \\ Relationship Between Autistic Traits, Social Problem-Solving Deficiencies, and De- pressive - Anxiety Symptoms: A Structural Equation Modeling Approach
}

\section{Ali Ebrahimi ${ }^{1}$, ${ }^{*}$ Abbas Ramezani Farani ${ }^{2}$, Mojgan Lotfi ${ }^{2}$, Mitra Hakim Shooshtari ${ }^{3}$}

1. MSc. Student, Department of Clinical Psychology, School of Behavioral Sciences and Mental Health (Tehran Institute of Psychiatry), Iran University of Medical Sciences, Tehran, Iran.

2. PhD in Clinical Psychology, Assistant Professor, Department of Clinical Psychology, School of Behavioral Sciences and Mental Health (Tehran Institute of Psychiatry), Iran University of Medical Sciences, Tehran, Iran.

3. Child and Adolescents Psychiatrist, Assistant Professor, Mental Health Research Center, School of Behavioral Sciences and Mental Health (Tehran Institute of Psychiatry), Iran University of Medical Sciences, Tehran, Iran.

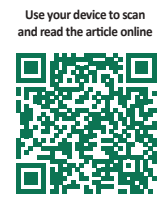

Cftation: Ebrahimi A, Ramezani Farani A, Lotfi M, Hakim Shooshtari M. [Relationship Between Autistic Traits, Social Problem-Solving Deficiencies, and Depressive - Anxiety Symptoms: A Structural Equation Modeling Approach (Persian)]. Iranian Journal of Psychiatry and Clinical Psychology. 2017; 23(3):278-293. https://doi.org/10.29252/nirp.ijpcp.23.3.278

https://doi.org/10.29252/nirp.ijpcp.23.3.278

Received: 13 Aug. 2016 Accepted: 12 Feb. 2017

Key words: Autistic traits, Social problem-solving, Depression, Anxiety, Structural equation modeling

\begin{abstract}
AB STRACT
Objectives The current study investigated the structural relationship between autistic traits, the components of social problem-solving deficiencies and depressive-anxiety symptoms.

Methods The study was conducted in 2015-2016 consisting of 336 students (male and female) with their age ranging from 18 to 35 years old. They were selected by using the convenience sampling method, and were assessed on Autism Spectrum Quotient (AQ), social problem-solving (SPSI-SF), and depression and anxiety symptoms (BAI and BDI-II). For data analysis, Pearson conduct coefficient, structural equation modeling, and boot stooping methods were conducted.

ResultsThe results of the analysis revealed that the deficit in social problem-solving component has an intermediary role in assessing the relationship between the severity of autistic traits, and depressionanxiety symptoms. All other hypothetical fitness indices model were in the appropriate range of positive cross-correlation $(0.45 \geq r \geq 0.25)$ between autistic traits, problem-solving deficiencies component, and depression- anxiety, All relations were found to be statistically significant $(P<0.01)$.

Conclusion The findings from the current study provide further support to the structural relationship between the components of the problem-solving deficiencies, severity of depressive - anxiety symptoms, and autistic traits. The understanding of how they are related to depressive - anxiety symptoms will hopefully help in informing about the future attempts for adopting an effective problem-solving programs for individuals with autistic traits.
\end{abstract}

\section{Extended Abstract}

\section{Introduction}

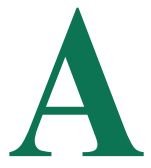

ccording to the current DSM-5 diagnostic criteria, Autism Spectrum Disorder (ASD) is characterized by a wide range of impairments in social communication, reciprocal interaction, and patterns of restricted and
Autism was formerly considered as a disorder, but recent evidences suggest that autistic traits are distributed among the general population, and they may exhibit autistic traits at sub clinical levels.

Individuals with autistic traits not only show the cognitive and behavioral characteristics of people with autism spectrum disorder but are also vulnerable to psychiatric and psychological problems. Evidence suggest that about circumscribed behaviors and interests.

\section{* Corresponding Author:}

Abbas Ramezani Farani, PhD

Address: Department of Clinical Psychology, School of Behavioral Sciences and Mental Health (Tehran Institute of Psychiatry), Iran University of Medical Sciences, Tehran, Iran.

Tel: +98 (21) 66506853

E-mail: ramezanifarani@gmail.com 
$65-80 \%$ of people with autism traits are misdiagnosed of other psychiatric illness.

Typical abnormalities are associated with autistic traits, including mood disorders, anxiety, specific phobias, obsessive-compulsive disorder, hyperactivity disorder, and attention deficit hyperactivity disorder. Studies have shown that, among psychological disorders in the subjects with autistic traits, depression and anxiety disorder are the most contributors.

The defect in the social problem-solving components is a vulnerable factor to the symptoms of depression and anxiety in different populations, so it is a question whether the defect in the social problem-solving components in individuals with autistic traits can make these individuals vulnerable to symptoms of depression and anxiety? There are few findings that examine the relationship between the social problemsolving problems in people with autistic traits and their effect on the level of vulnerability to depression and anxiety in individuals with autistic traits.

\section{Methods}

The participants for this study were 336 young-adult students recruited from the University of Tehran in Iran. The sample included 191 men (56.8\%) and 145 women (43.2\%) with age ranging between 18 and $35(\mathrm{M}=22.66, \mathrm{SD}=3.65)$. All participants gave their informed consent for this study, and the ethical approval was received from the Iran University of Medical Ethical Committee.

\section{Materials}

\section{Autism-Spectrum Quotient}

Baron-Cohen and colleagues designed a self-report $\mathrm{Au}-$ tism-Spectrum Quotient questionnaire (AQ50) to measure the Autistic Trait consisting of 50 questions that measure
5 areas of autistic traits, including social skills, attention change, communication, attention to detail and imagination, and covers every 10 questions in one of the domains. The AQ has reasonably good psychometric properties with reported internal reliability values of 0.67 and 0.82 . The testretest reliability of the Persian version of AQ50 $(r=0.82)$ and internal consistency $(\mathrm{a}=0.76)$ was reported within the threeweek study.

Social Problem-Solving Inventory-Revised: Short Form

The Social Problem-Solving Inventory - Revised: Short (SPSI-R:S) Form is a 25-itemed self-report instrument that evaluates the characteristics of social problem-solving, including problem-solving orientation and performance. Internal consistency and test-retest coefficients of Persian SPSI$\mathrm{R}: \mathrm{S}$ were 0.86 and 0.65 , respectively.

\section{Beck' Depression Inventory- Second Edition (BDI-II)}

The BDI-II is one of the most widely used self-report instruments for assessing the symptoms of depression. The Persian version of BDI-II showed a high internal consistency $(\mathrm{a}=0.87)$ and acceptable test-retest reliability $(\mathrm{r}=0.74)$.

\section{Beck Anxiety Inventory (BAI)}

The BAI is a 21-itemed self-report questionnaire, which is designed to assess the presence and degree of anxiety symptoms within the past week and distinguish them from those of depressive symptoms. Persian version of BAI showed good reliability $(\mathrm{r}=0.72)$ and validity $(\mathrm{r}=0.83)$.

\section{Results}

\section{Preliminary analyses and descriptive statistics}

Across all participants, the mean score for the total assessment was $\mathrm{AQ}=20.75$ ( $\mathrm{SD}=4.55$, range: $11-34)$, SPSI-S:

Table 1. Means, standard deviations, and intercorrelations for all variables $(\mathrm{N}=336)$

\begin{tabular}{|c|c|c|c|c|}
\hline Variables & 1 & 2 & 3 & 4 \\
\hline 1. Autistic traits & 1 & & & \\
\hline 2. Social problem-solving & $0.26^{* *}$ & 1 & & \\
\hline 3. Anxiety symptoms & $0.25^{* *}$ & $0.26^{* *}$ & 1 & \\
\hline 4. Depressive symptoms & $0.34^{* *}$ & $0.28^{* *}$ & $0.45^{* *}$ & 1 \\
\hline Means & 20.75 & 42 & 10.17 & 11.82 \\
\hline Standard deviations & 4.45 & 7.77 & 7.66 & 8.28 \\
\hline
\end{tabular}


Table 2. Measures of goodness of fit of measurement model

\begin{tabular}{ccccccccccc}
\hline Index & Chi- Square & df & $\mathbf{P}$ & RMSEA & RMR & CFI & GFI & IFI & NFI & NNFI \\
\hline Value & 97.21 & 38 & 0.000 & 0.06 & 0.05 & 0.96 & 0.95 & 0.95 & 0.94 & 0.95 \\
\hline
\end{tabular}

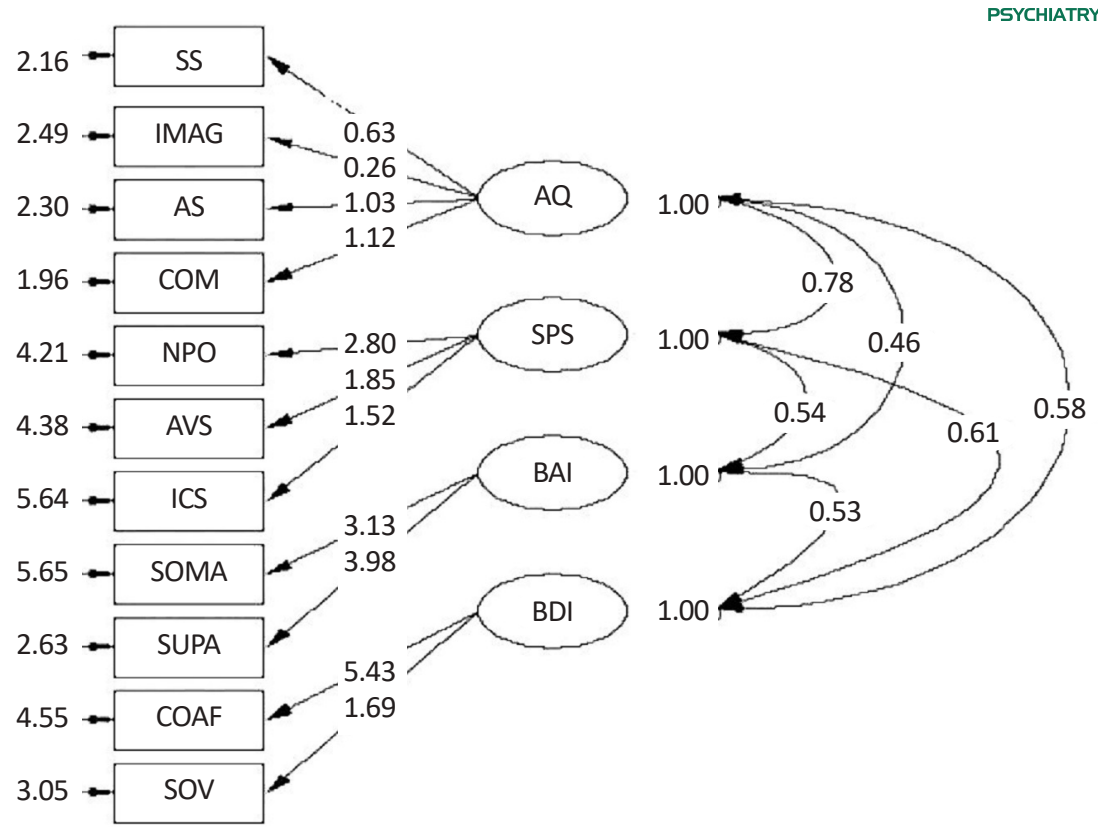

Figure 1. Measurement model

AQ: Autism-Spectrum Quotient; SPS: Social Problem Solving; BAI: Beck’s depression Inventory; BDI-II: Beck’s Anxiety Inventory; SS: Social Skill; IMAG: Imagination; AS: Attention Switching; COM: Communication; NPO: Negative Problem Solving Orientation; AVS: Avoidance Style; ICS: Impulsivity/Carelessness Style; SOMA: Somatic Symptoms; SUPA: Subjective anxiety and Panic symptom; COAF: Cognitive-Affective; SOV: Somatic-Vegetative

$\mathrm{F}=42(\mathrm{SD}=7.77$, range: $16-65), \mathrm{BAI}=42(\mathrm{SD}=7.66$, range: $0-42$ ), and $\mathrm{BDI}-\mathrm{II}=11.82$ ( $\mathrm{SD}=8.28$, range: $0-39$ ). The means, standard deviations, and intercorrelations for all variables can be viewed in Table 1 .

\section{Structural equation modeling analysis}

The confirmatory factors supported the factor structure for AQ, SPSI, BDI-II, and BAI factors used in the current analyses, but the standardized coefficient of attention to detail (ATD) was not significant. Therefore, ATD factor was removed, and the measurement model was again evaluated. Fit indices (Table 2) demonstrated a good fit suggesting that this measurement model offers an acceptable representation of the latent variables (Figure 1).

Fit indices demonstrated (Table 3) the significance $(\mathrm{P}<0.01)$ of the model's good fit suggesting that this structural equation modeling (SEM) approaches (Figure 2) support the relationship between autistic traits, problem-solving deficits, depression, and anxiety symptoms. The structural equation modeling along with standardized coefficients (Figure 2) suggested the autistic traits as an exogenous variable, which affected the problem-solving deficient components (Standard coefficient $=0.81$ ). Furthermore, problem-solving

Table 3. Measures of goodness of fit of structural equation modeling

\begin{tabular}{ccccccccccc}
\hline Index & Chi- Square & df & $\mathbf{P}$ & RMSEA & RMR & CFI & GFI & IFI & NFI & NNFI \\
\hline Value & 115.28 & 41 & 0.000 & 0.07 & 0.05 & 0.95 & 0.94 & 0.95 & 0.93 & 0.94 \\
\hline
\end{tabular}




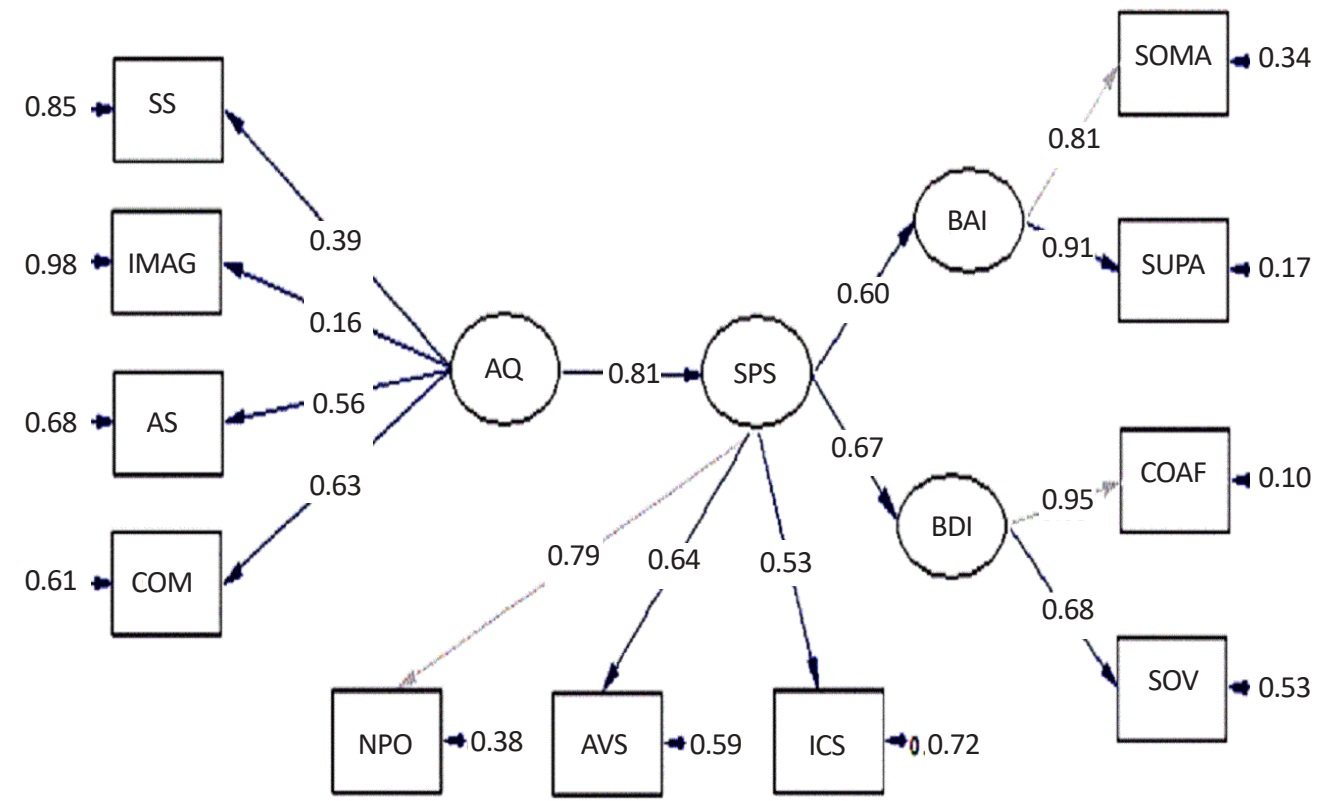

Figure 2. structural equation modeling

Table 4. Standard coefficient and T-Value of observed variables

\begin{tabular}{rccc}
\hline & Observed Variables & Standard Coefficient & T \\
\hline & SS & $0.39^{* *}$ & 6.25 \\
AQ & IMAG & $0.16^{* *}$ & 2.56 \\
& AS & $0.56^{* *}$ & 9.07 \\
& COM & $0.63^{* *}$ & 10.03 \\
SPSI & NPO & $0.81^{* *}$ & 15.32 \\
& AVS & $0.66^{* *}$ & 12.22 \\
BAI & ICS & $0.54^{* *}$ & 9.57 \\
& SOMA & $0.80^{* *}$ & 14.92 \\
BDI-II & SUPA & $0.93^{* *}$ & 17.52 \\
& COAF & $0.93^{* *}$ & 16.95 \\
\hline
\end{tabular}

$* * \mathrm{P}<0.01$

deficient components effected on depression (Standard coefficient $=0.67$ ) and anxiety (Standard coefficient $=0.60$ ). Ineffective social problem-solving components (Avoidance style, Impulsive/Carelessness Style, and Negative Problem Orientation) result in depression and anxiety symptoms of autistic traits (Table 4).

\section{Conclusion}

The current study aimed to investigate the Structural Equation Modeling (SEM) analysis of the relationship between
Iranian Journal of

PSYCHIATRY AND CUNICAL PSYCHOLOGY

autistic traits, social problem-solving deficient components, and depression as well as anxiety symptoms in an Iranian student sample. Findings of the current study are in consistent with Rosbrook and Whittingham (2010) [20], Zarekar et al., (2014) [35], Liew et al., (2015) [36], and Jackson and Dritschel (2016) [13]. Furthermore, Our model was statistically confirmed.

According to the D'Zurilla \& Nezu model, the social problem-solving components act as indicators of individual adequacy, and thus, can be considered as a sign of quality of life 
in interpersonal interactions. Individuals with autistic traits are prone to experience the symptoms of anxiety and depression during the situations of problem-solving, as a result of which they use inefficient problem-solving styles (such as negative-orientation toward the problem, avoidance style, and impulsivity/inclination style).

The social performance of people with autistic traits shows that social disruptions in them can be rooted in the perception of social inefficiency and weak self-efficacy, which gets reflected by their negative orientation of problem-solving in problematic situations. In addition, the results of decisionmaking investigations suggest that individuals with autistic traits are heavily distracting from the risk, which is evident from their avoidance style further leading to vulnerability to anxiety symptoms. Ultimately, comorbid Attention Deficit Hyperactivity Disorder (ADHD) in people with autistic traits, especially exhibiting a high degree of inhibition / inhibition symptoms, may make them susceptible to impulsive / unconscious style.

According to our knowledge, this study can be considered as an elementary and important study on the role of the mediating the defects in the social problem-solving components to assess the relationship between autistic traits and symptoms of depression and anxiety by using structural equations modeling. This study also has some limitations. Given that all participants of the current study were university students, therefore the results of this study are limited to student sampling method. Although SEM approach provides the possibility to test complex hypotheses, cross-sectional nature of this study precludes the causal conclusions and accurate understanding of the true nature of these relationships. Finally, only self-report instruments were used to evaluate each of the variables in this study. It is, therefore, suggested that future research should use a variety of measurement techniques to assess the social problem-solving components.

\section{Ethical Considerations}

\section{Compliance With Ethical Guideline}

The consent was taken from the participants and the research objectives were explained and the confidentiality of the participants' information was assured.

\section{Funding}

This article is based on the first author MSc. thesis in the Department of Clinical Psychology, School of Behavioral Sciences and Mental Health (Tehran Institute of Psychiatry), Iran University of Medical Sciences.

\section{Conflict of Interest}

The authors declare no conflicts of interest.

\section{Acknowledgements}

We acknowledge the participants of this research. 


\title{
ارتباط ميان صفات اتيستيك، نقص مؤلفهاهاى حل مسئله اجتماعى، نشانهایى افسردتى و اضطراب:

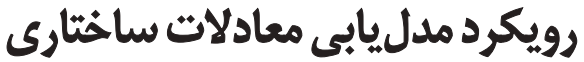

\author{
على ابراهيمى'? "عباس رمضانى فرانى'، مزّان لطفى'، ميترا حكيم شوشترى"
}

ا - دانشجوى كارشئاسى ارشده كروه روانشئاسى بالينى، دانشكده علوم رفتارى و سلامت روان (انستيتو روانيزشكى تهران)، دانشكاه علوم يزشكى و خدمات بهاشتى درمانى ائيرانه، تيهرانْ، ايران.

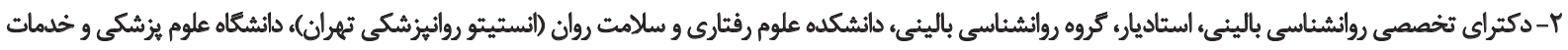

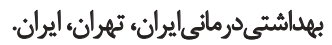

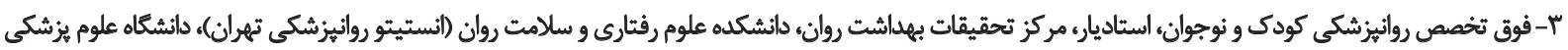

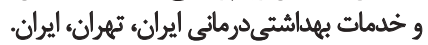

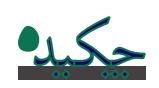

الهدأك هدف مطالعه حاضر بررسى روابط ساختًارى ميان صفات اتيستيك، نقص مؤلفههاى حل مسئله اجتماعى، نشانهاى افسردكى و اضطراب

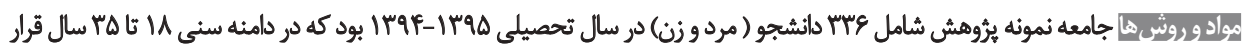

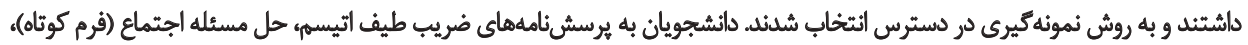

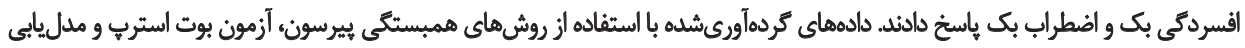

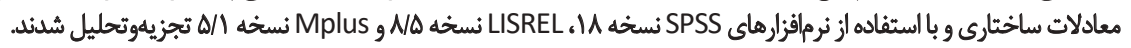

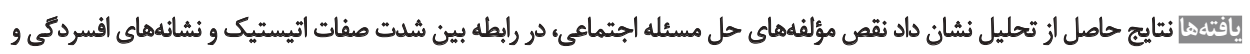

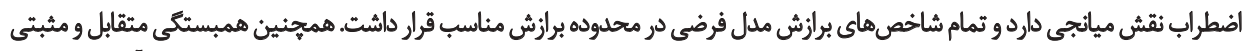

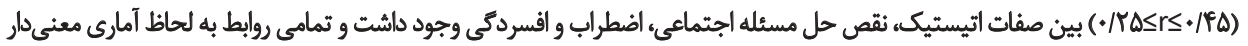

بون (P</.1)

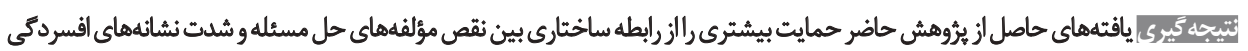

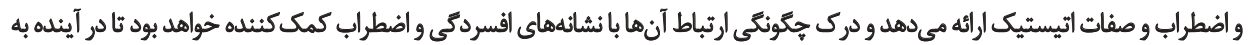

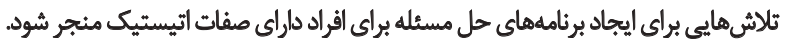

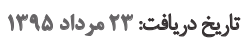

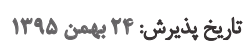

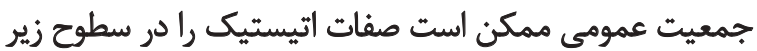

daies

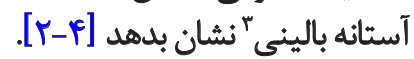

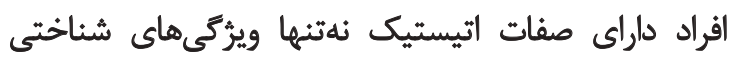

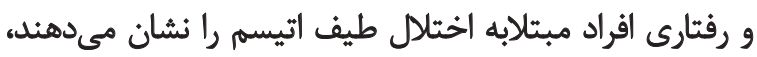

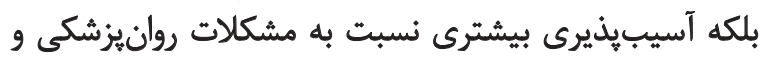

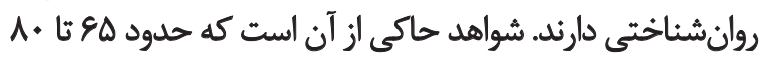

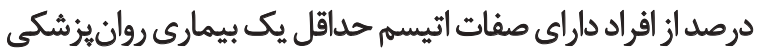

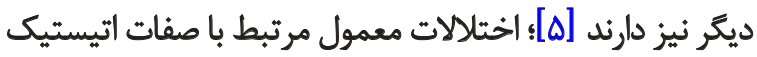

كليدوارهها:

صفات اتيستيك، حل

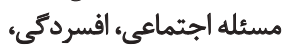

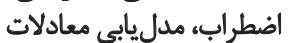
ساختيارى

اختلال طيف اتيسم' نوعى اختلال رشد عصبى مادامالعمر

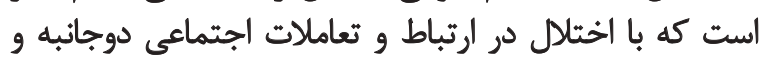

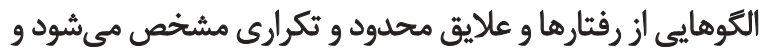

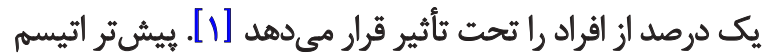

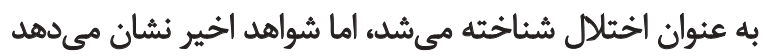

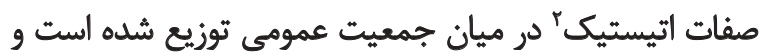

\section{Autism Spectrum Disorder}

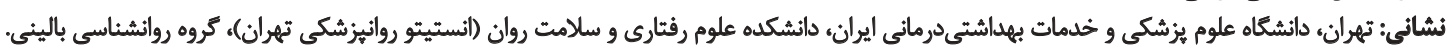
ت تلفن:

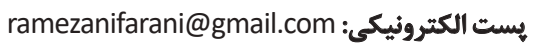




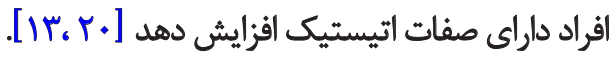
با جمعبندى مطالب ذكرشده به نظر ميرسد افراد در تمام

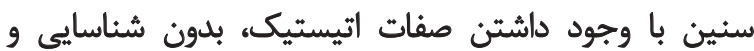

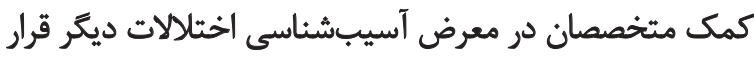

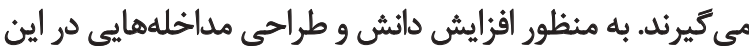

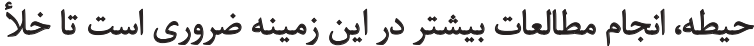

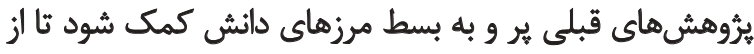
اين طريق دانش ما در اين زمينه افزايش يابد و درد اين اين حيطه

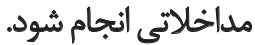

روش يُوهش حاضر بيمايشى و از نوع همبستخى است. جامعه

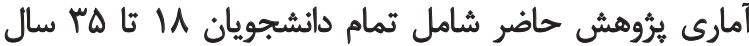

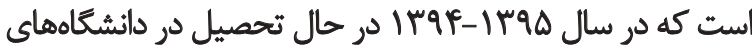

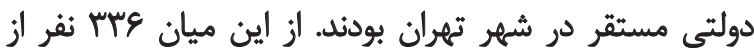

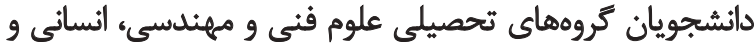

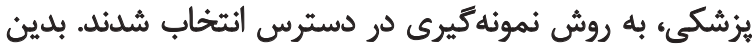

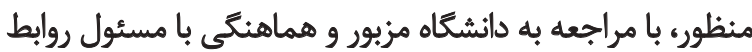

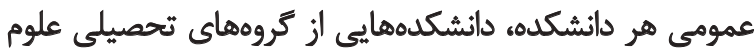

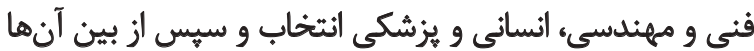

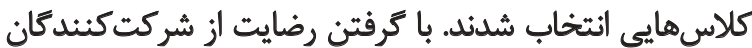

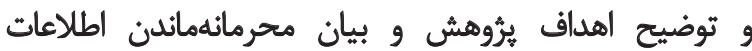

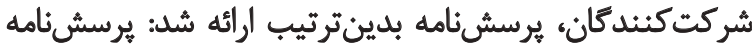

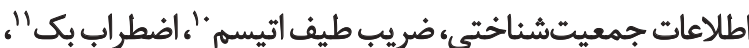

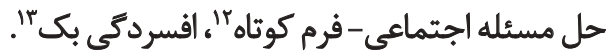

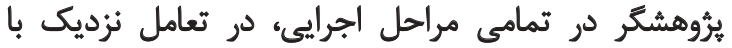

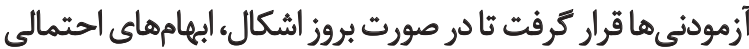

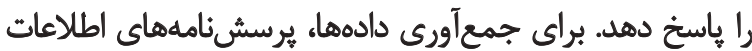

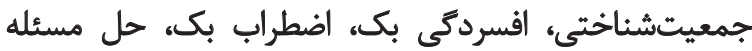

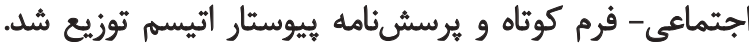

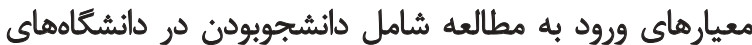

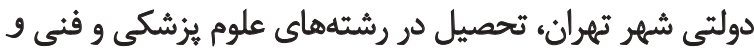

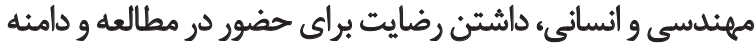

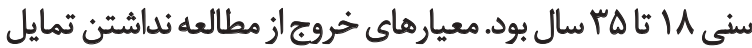

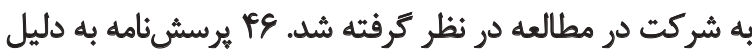

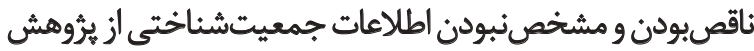

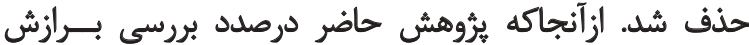

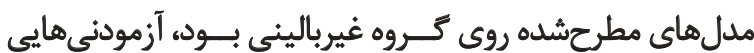

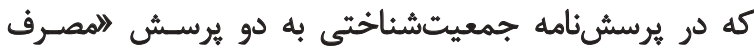

10. Autism Spectrum Quotient

11. Beck Anxiety Inventory

12. Social Problem Solving Inventory- Short Form

13. Beck Depression Inventory

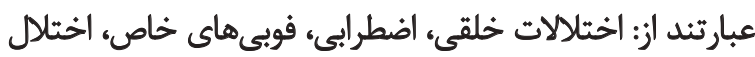

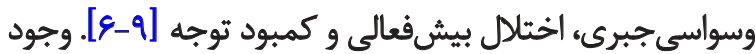

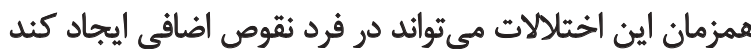

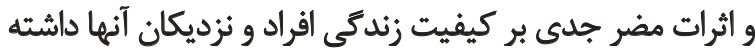

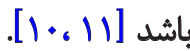

مطالعات ئشانكًر اين است كه در ميان اختلالات روانشناختى در

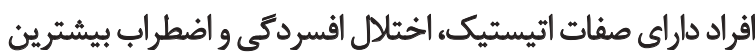

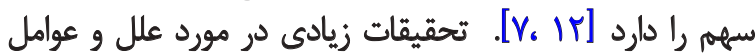

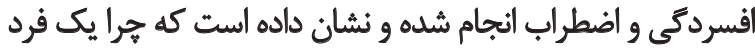

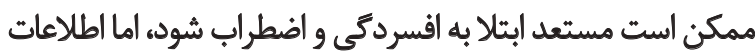

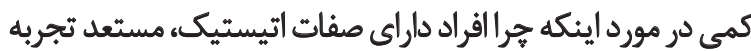

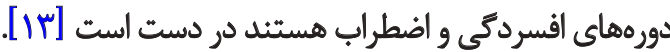

توانايىهاى ضعيف حل مسئله اجتماعى افراد را در برابر

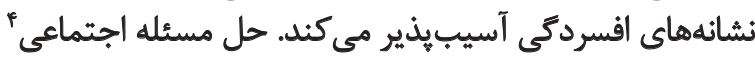

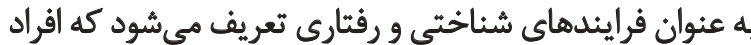

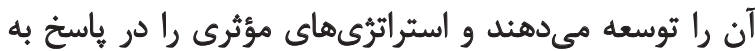

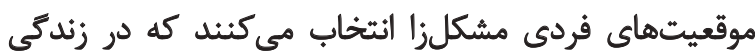

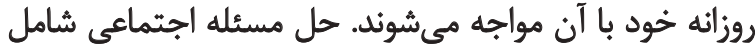

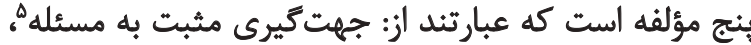

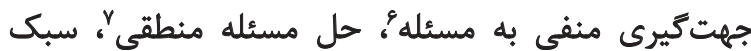

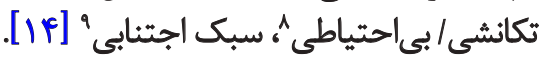

اولين مطالعات در شناسايي نقش كليدى نقص حل مسئله

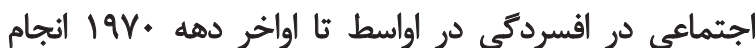

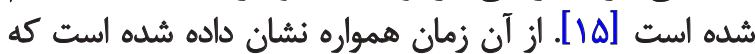

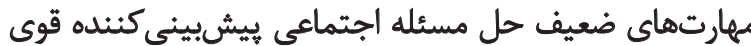

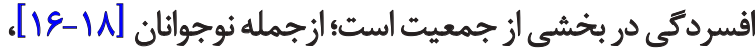

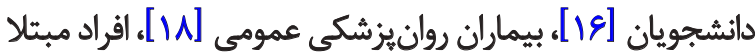
به اختلال اضطراب [1ه ] و بيماران افسرده بالينى [19] [19. نقص در مؤلفههاي حل مسئله اجتماعى عامل آسيبيذيرى

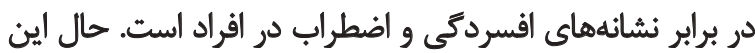

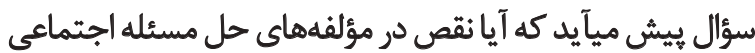

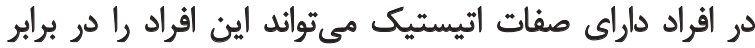

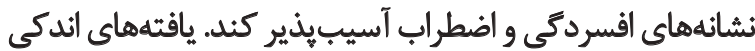

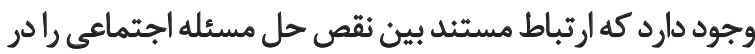

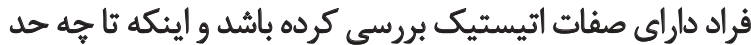

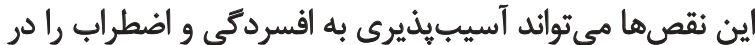

4. Social Problem Solving

5. Positive Problem Solving Orientation

6. Negative Problem Solving Orientation

7. Rational Problem Solving

8. Impulsivity/ Carelessness Style

9. Avoidance Style 
عاطفى و رفتارى به موقعيتهاي حل مسئلهاى در زندكى واقعى،

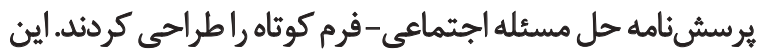

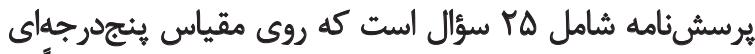

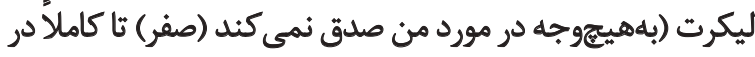

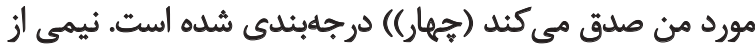

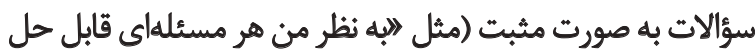

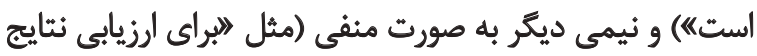

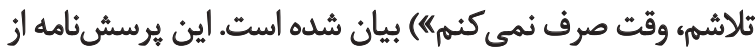

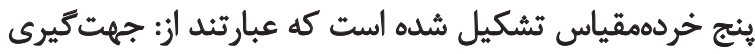

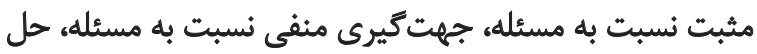

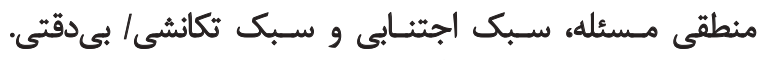

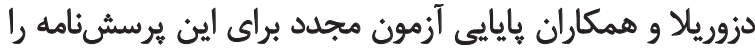

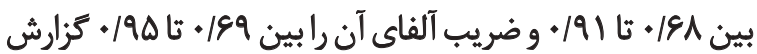

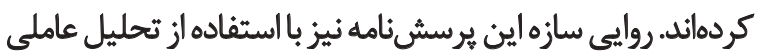

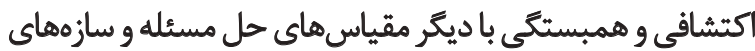
روانشناختى هميوش تأييد شده است.

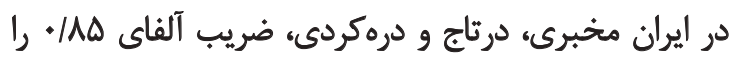

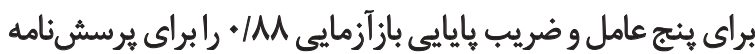

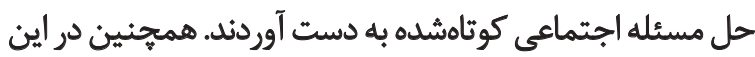

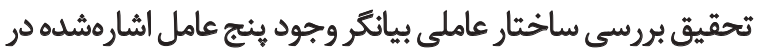

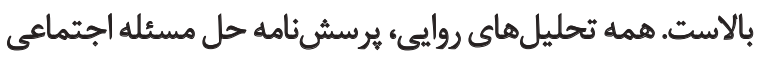

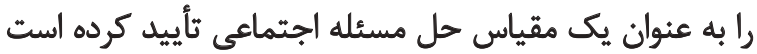

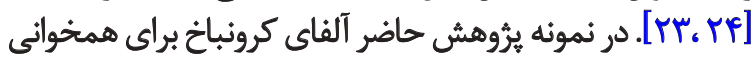

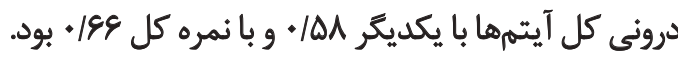

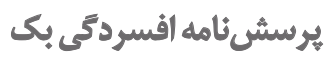

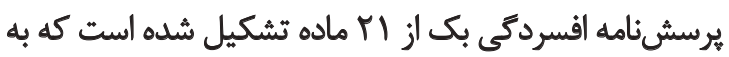

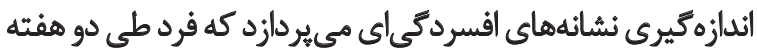

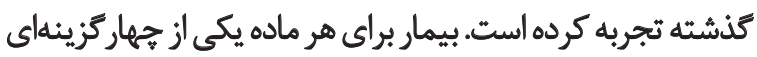

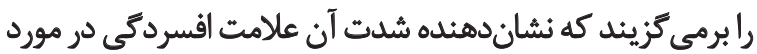

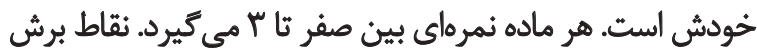

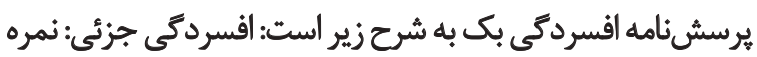

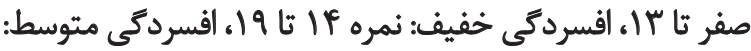

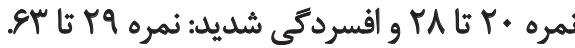

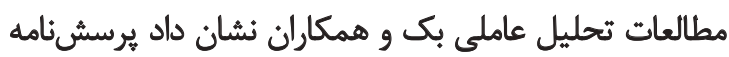

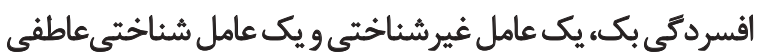

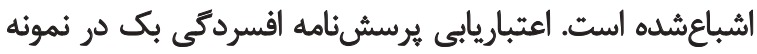

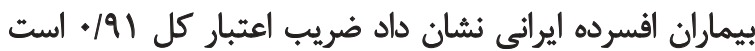

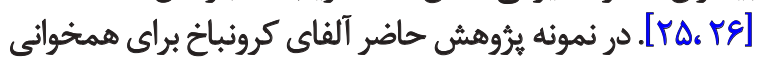

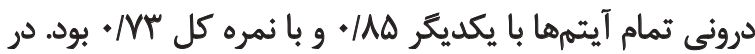

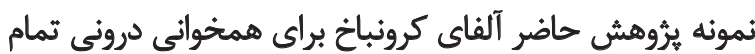

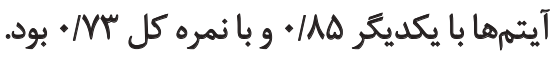

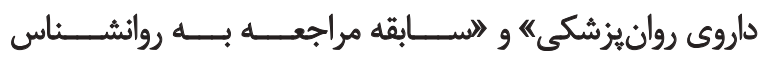

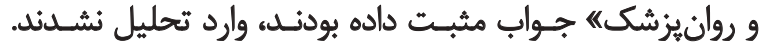

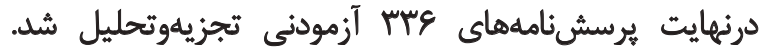

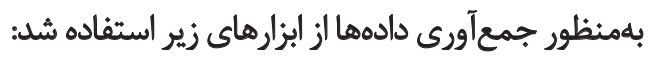

$$
\text { يرسشنامه ضريب طيف اتيسم }
$$

بارون كوهن و همكاران به منظور جمع آورى دادههاى مربوط إنيف

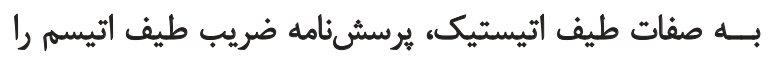

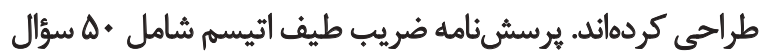

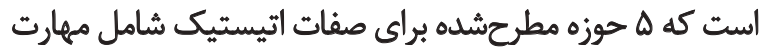

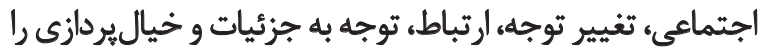

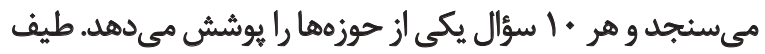

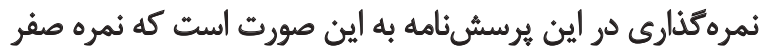

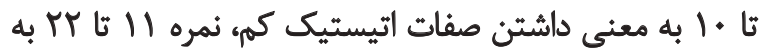

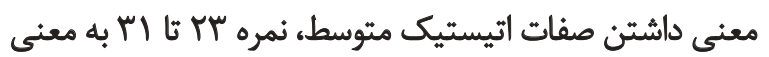

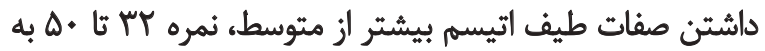

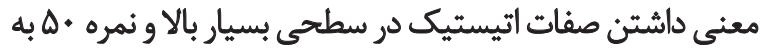
معنى داشتن بيشترين صفات اتيستيك است آتيكي

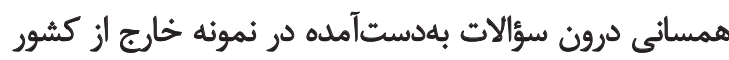

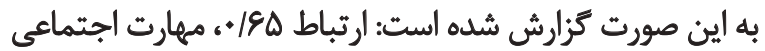

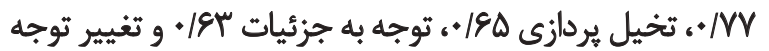
19V

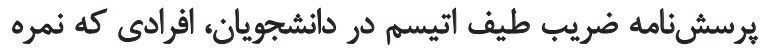

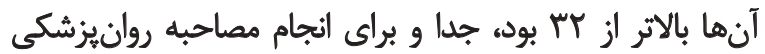

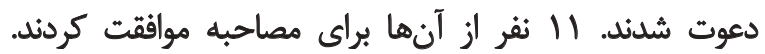

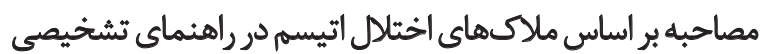

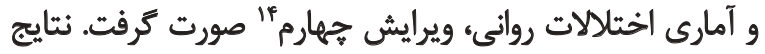

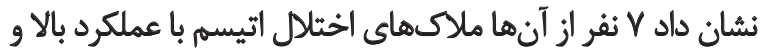

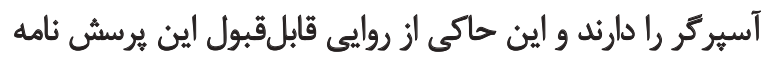

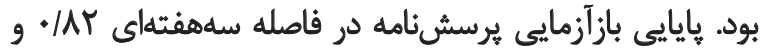

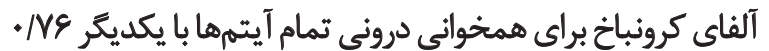

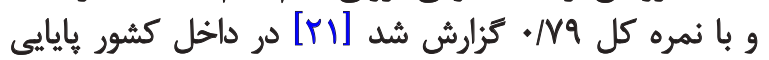

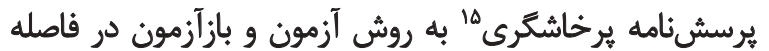

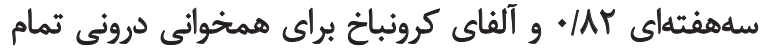

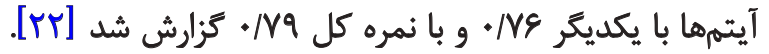

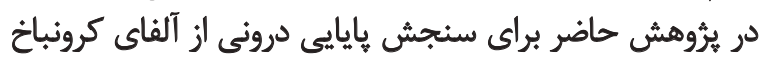

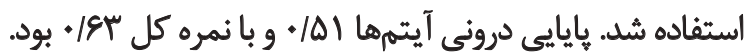

$$
\text { برسشئامه حل مسئله اجتماعى - فرم كوثاه }
$$

دزوريلاو همكاران به منظور اندازمكيرى ياسخهاى شناختى،

14. Diagnostic and Statistical Manual of Mental Disorders, Fourth Edition (DSM-IV)

15. Aggression Questionnaire (AQ) 


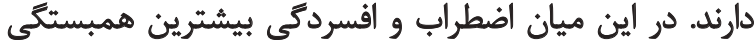

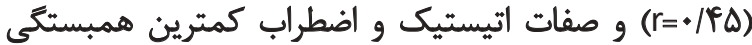

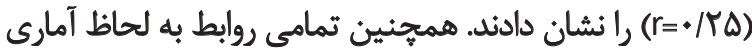

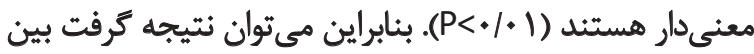

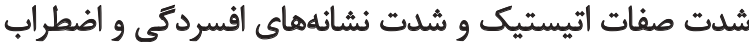
رابطه مثبت و معنادارى وجود دارد. از آنجا كه استفاده از مدل معادلات ساختارى داراى

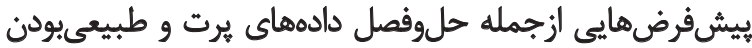

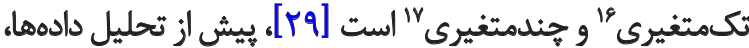

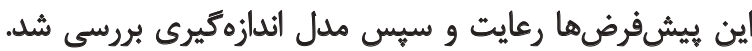

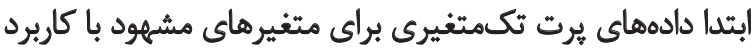

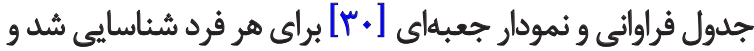

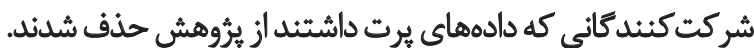

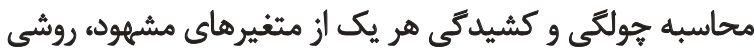

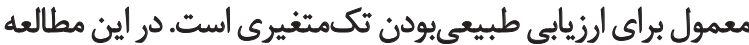

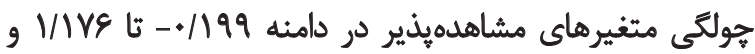

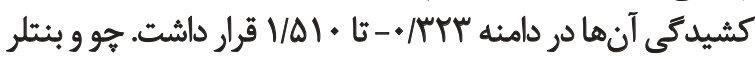

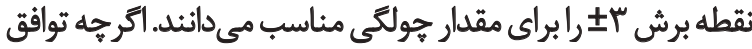

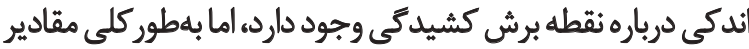

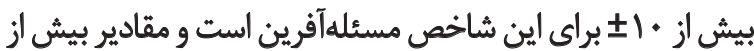

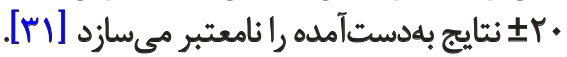

در اين مطالعه فرض طبيعىبودن جندمتئغيرى با محاسبه

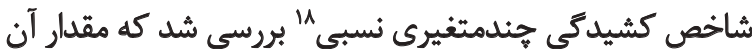

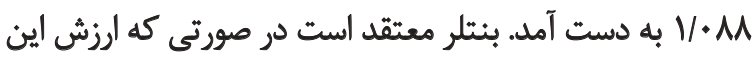

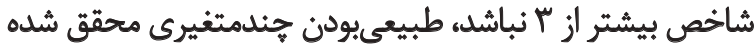

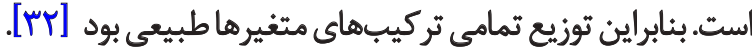
ييش از ارزيابى مدل ساختارى، به منظور صحت متغيرهاى إئاي

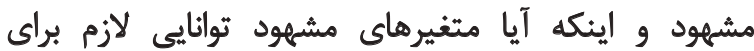

16. Univariate normality

17. Multivariate normality

18. Relative Multivariate Kurtosis

$$
\text { يرسش نامه الضطراب بك }
$$

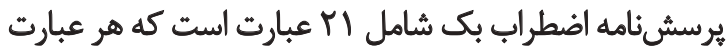

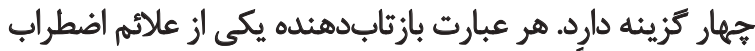

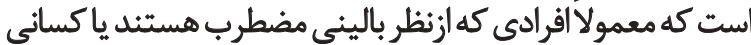

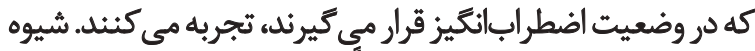

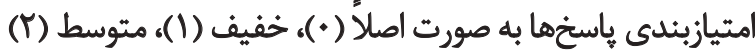

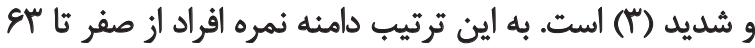

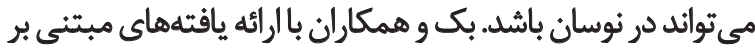

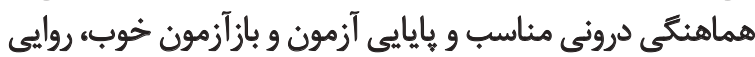

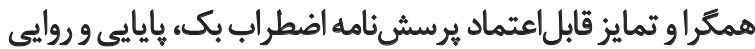

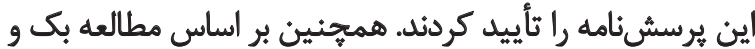

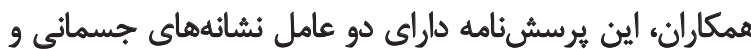

$$
\text { اضطراب ذهنى و يانيك است. }
$$

در ايران مطالعات كاويانى و همكاران نشان داد كه برسش بإنامه

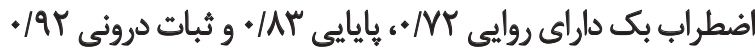

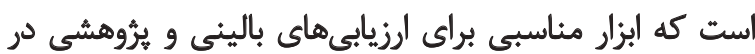

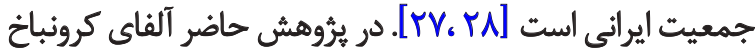

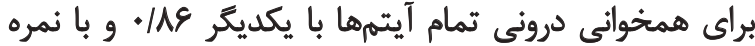

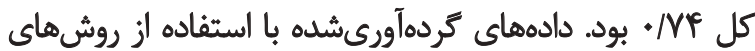

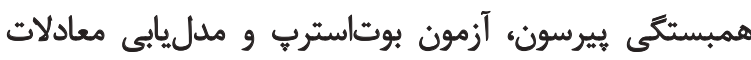

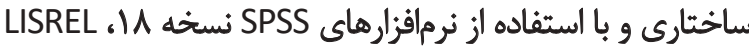
نسخه Mplus g/D نسخه

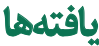

ميانكين و انحراف معيار سن آزمودنى هاى اين مطالعه به ترتيب

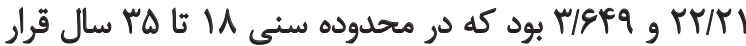

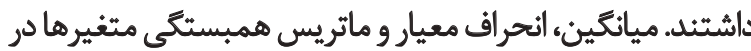

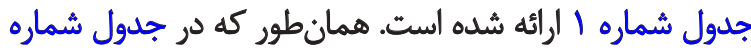

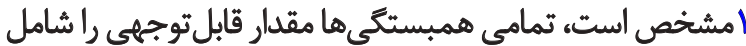

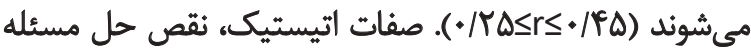

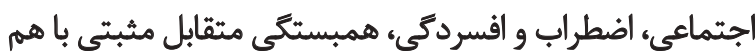

\begin{tabular}{|c|c|c|c|c|}
\hline$\varepsilon$ & $r$ & $r$ & 1 & متغيرها \\
\hline & & & 1 & 1. صفات اتيستيك \\
\hline & & 1 & 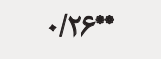 & Y. ثقص حل مسثله اجتماعى \\
\hline & 1 & "Kq" &.$/ T \omega^{* *}$ & ب. اضطراب \\
\hline 1 &.$/ P \phi^{* * *}$ & $\cdot / \Lambda^{* *}$ &.$/ m p e n$ & ب. ب. افسرد5ى \\
\hline W/Ar(NYA) & $1+/ N Y(Y / 99)$ & $P r(Y M V)$ & $r \cdot N \Delta(\varphi / P \Delta)$ & مياثكين (انحراف معيار) \\
\hline
\end{tabular}

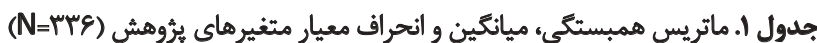


جدول r. شاخصهاى برازش مدل اندازهيرى

\begin{tabular}{|c|c|c|c|c|c|c|c|c|}
\hline IFI & NNFI & NFI & GFI & CFI & RMR & RMSEA & df & Chi-Square \\
\hline.$/ 9 \Delta$ & $+/ 9 \Delta$ &.$/ 94$ &.$/ 9 \Delta$ &.$/ Q 8$ & .1 .0 & .1 .8 & ए人 & QV/TI \\
\hline
\end{tabular}

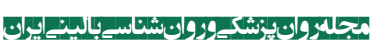

صفات اتيستيك به عنوان متغير برونزا با ضريب استاندارد

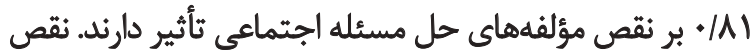

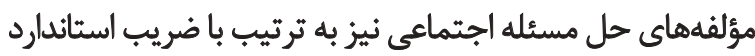

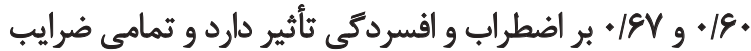

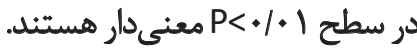

در مطالعه حاضر براي ارزيابى روابط واسطهاي از آزمون بوت

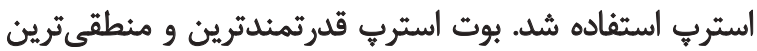

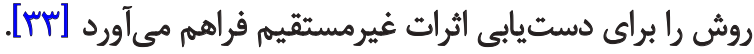

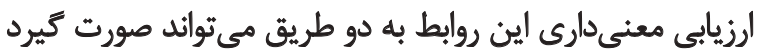

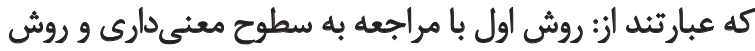

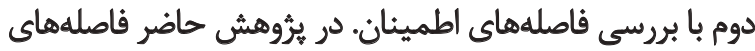

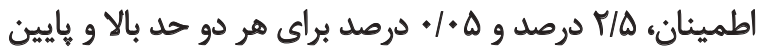

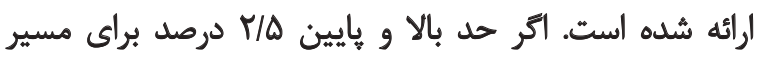

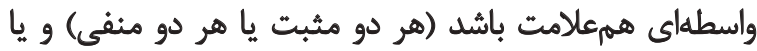

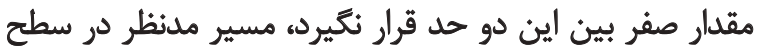

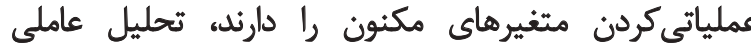

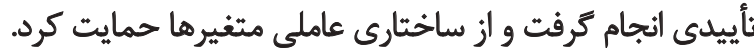
خردهمقياس لاتوجه به جزئيات

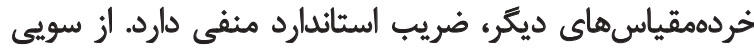

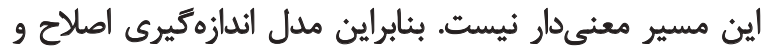

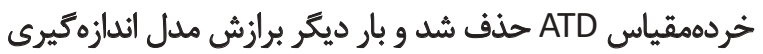

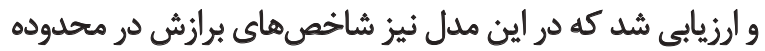

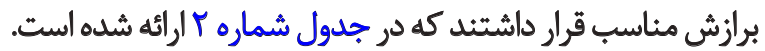
در مرحله بعد مدل ساختارى بررسى شد. همان گونه كه جدول

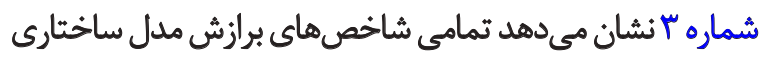

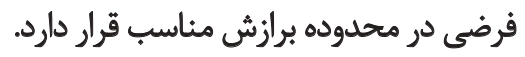
تصوير شماره r مدل ساختارى فرضي را به همراه ضرايب

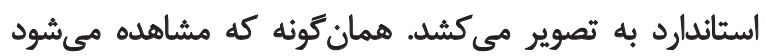

19. Attention to Detail (ATD)

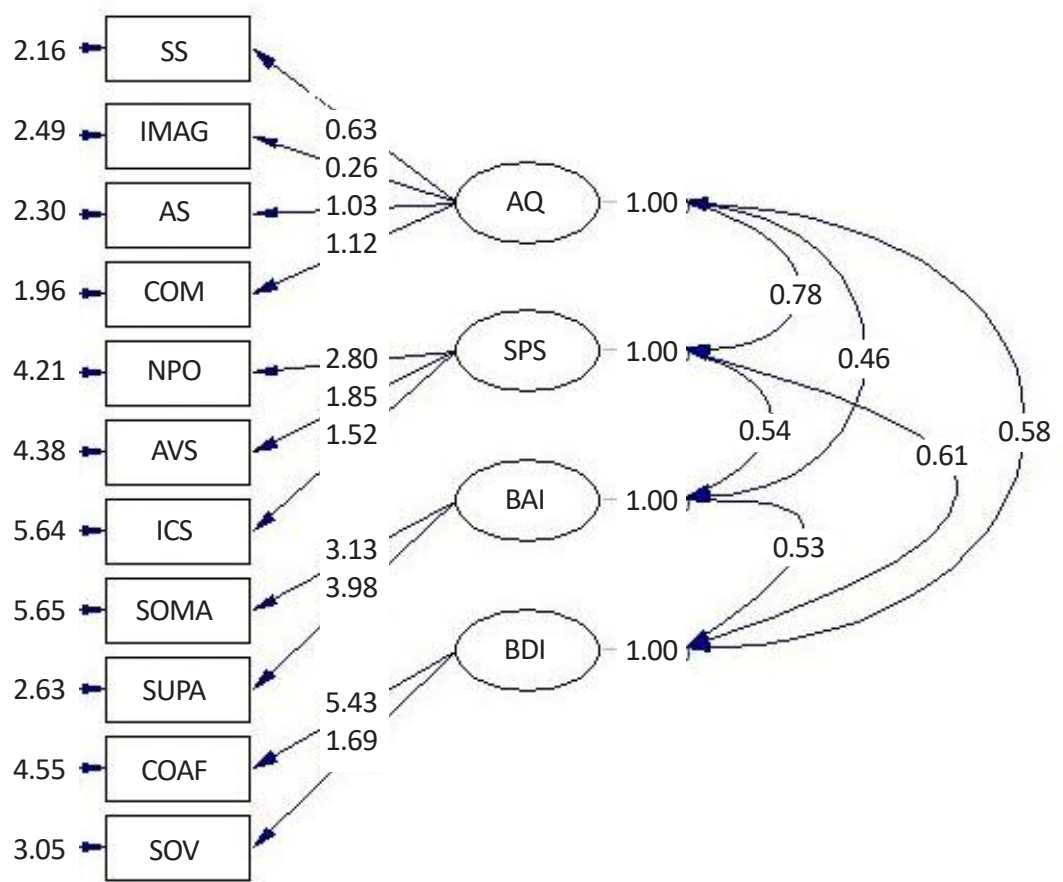

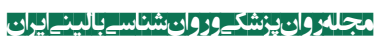

تصوير ا. مدل اندازهيرى ارتباط بين صفات اثيستيك، نقص مولفههاى حل مسئله اجتماعى، نشانههاى افسردكى و اضطراب

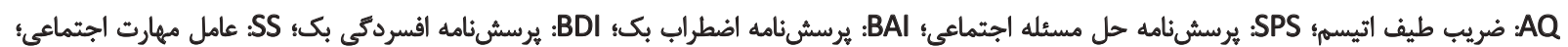

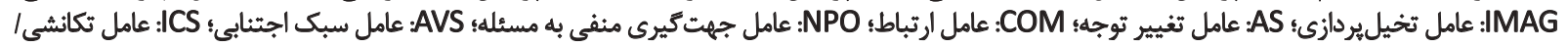

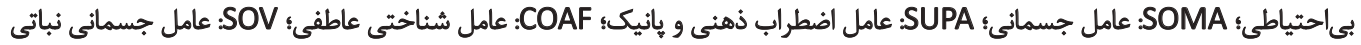


جدول ऍ. شاخصهاى برازش مدل ساختارى صفات اتيستيك، نقص مولفههاي حل مسئله اجتماعى، نشائهاي افسردكى و اضطراب

\begin{tabular}{llllllllll}
\hline RFI & IFI & NNFI & NFI & GFI & CFI & RMR & RMSEA & df & Chi-Square \\
\hline$* / 9)$ &.$/ 9 \Delta$ &.$/ 9 F$ &.$/ 9 \%$ &.$/ 9 F$ &.$/ 9 \Delta$ & $\% \Delta$ & $\% / \gamma$ & $\uparrow /$ & $11 \Delta / r \Lambda$ \\
\hline
\end{tabular}

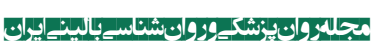

و صفات اتيستيك بودانداند طرح كلى مدل فرضى بر اين اساس

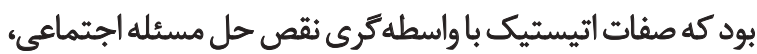

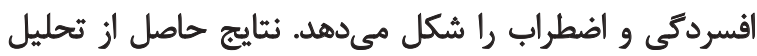

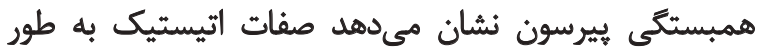

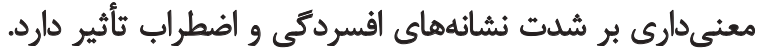

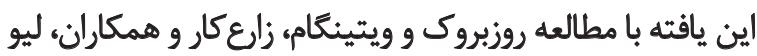

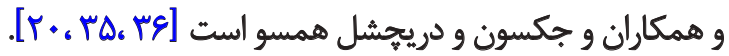
در تبيين اين يافتها بر اساس مدل حل مسئله اجتماعي

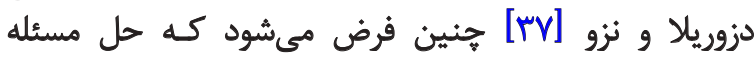

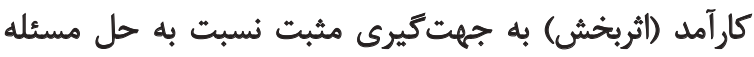

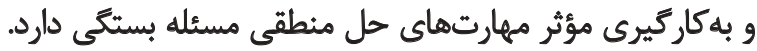

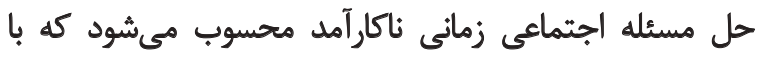

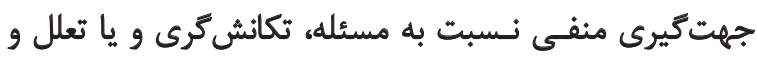

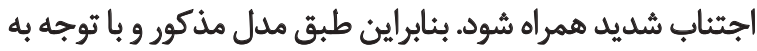

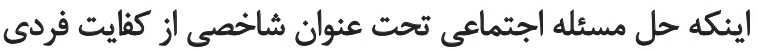

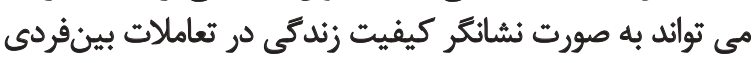

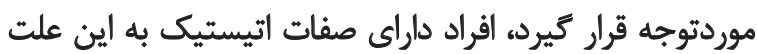

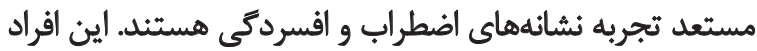

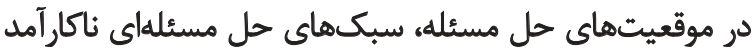
(مثل جهت كيرى منفى نسبت به مسئله، سبك اجتنابى و سبك ماكي

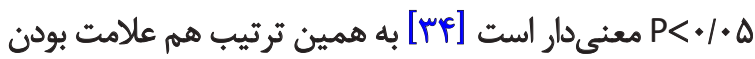

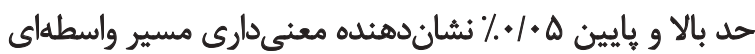

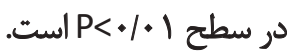

همان كونه كه جدول شماره f أشان مى دهد مسير صفات

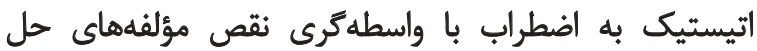

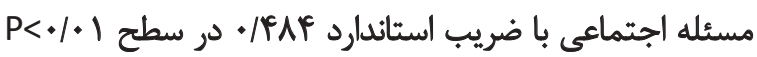

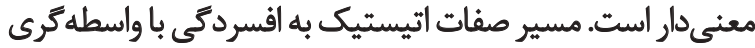

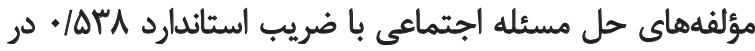

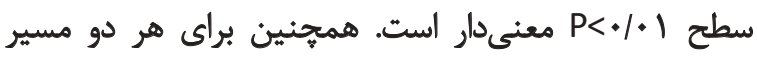

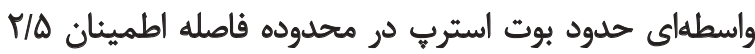
درصد و ه ٪ درصد همعلامت است. بحث مطالعه حاضر با هدف بررسى روابط ساختارى ميان صفات

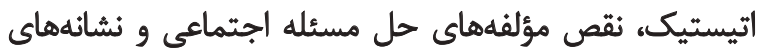

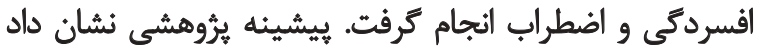

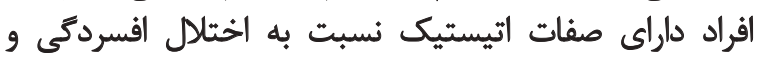

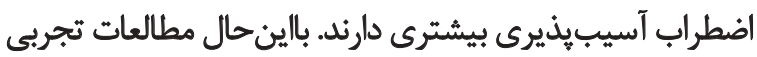
كمترى به دنبال يافتن رابطه تعاملى بين اين عوامل آسيبيدير مائير

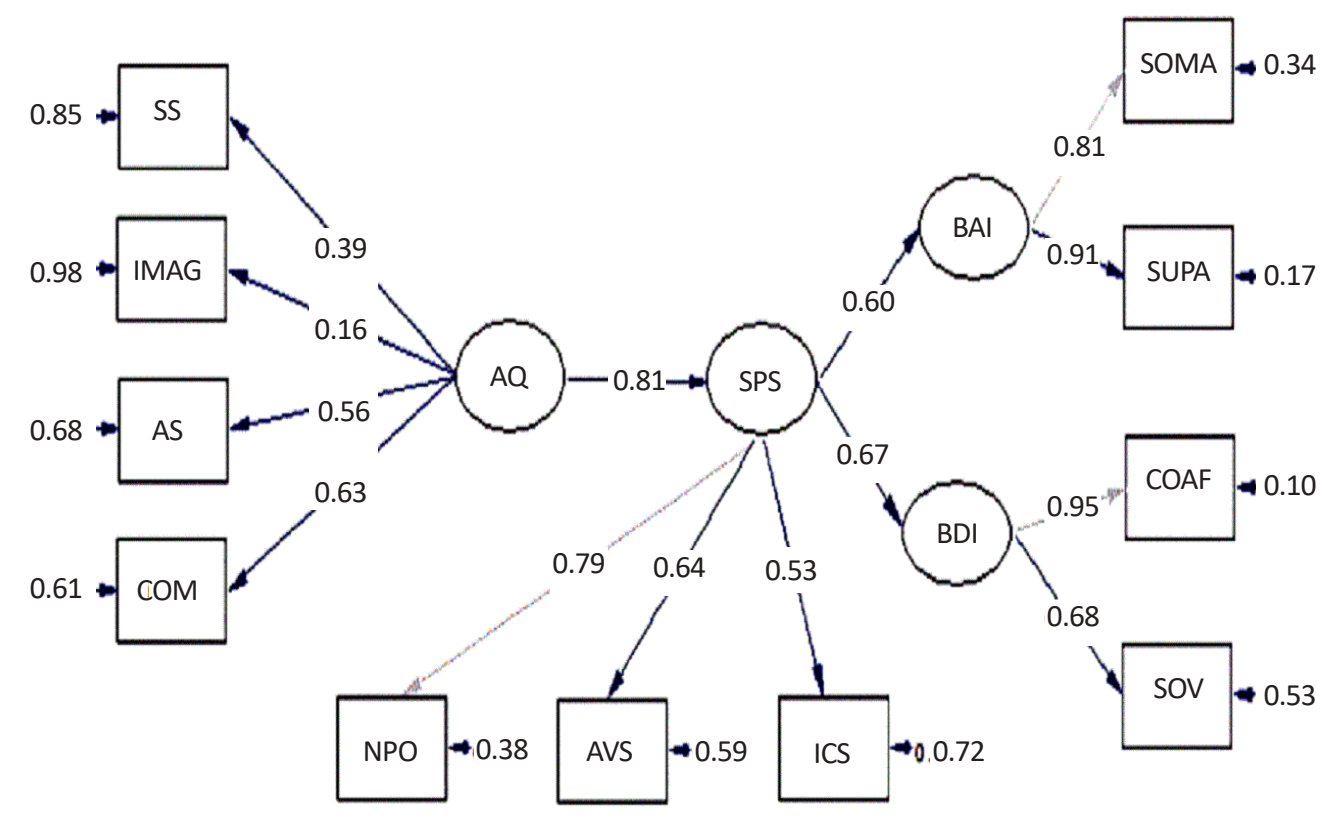


جدول F. نتايج آزمون بوت استرب براى روابط واسطهاى

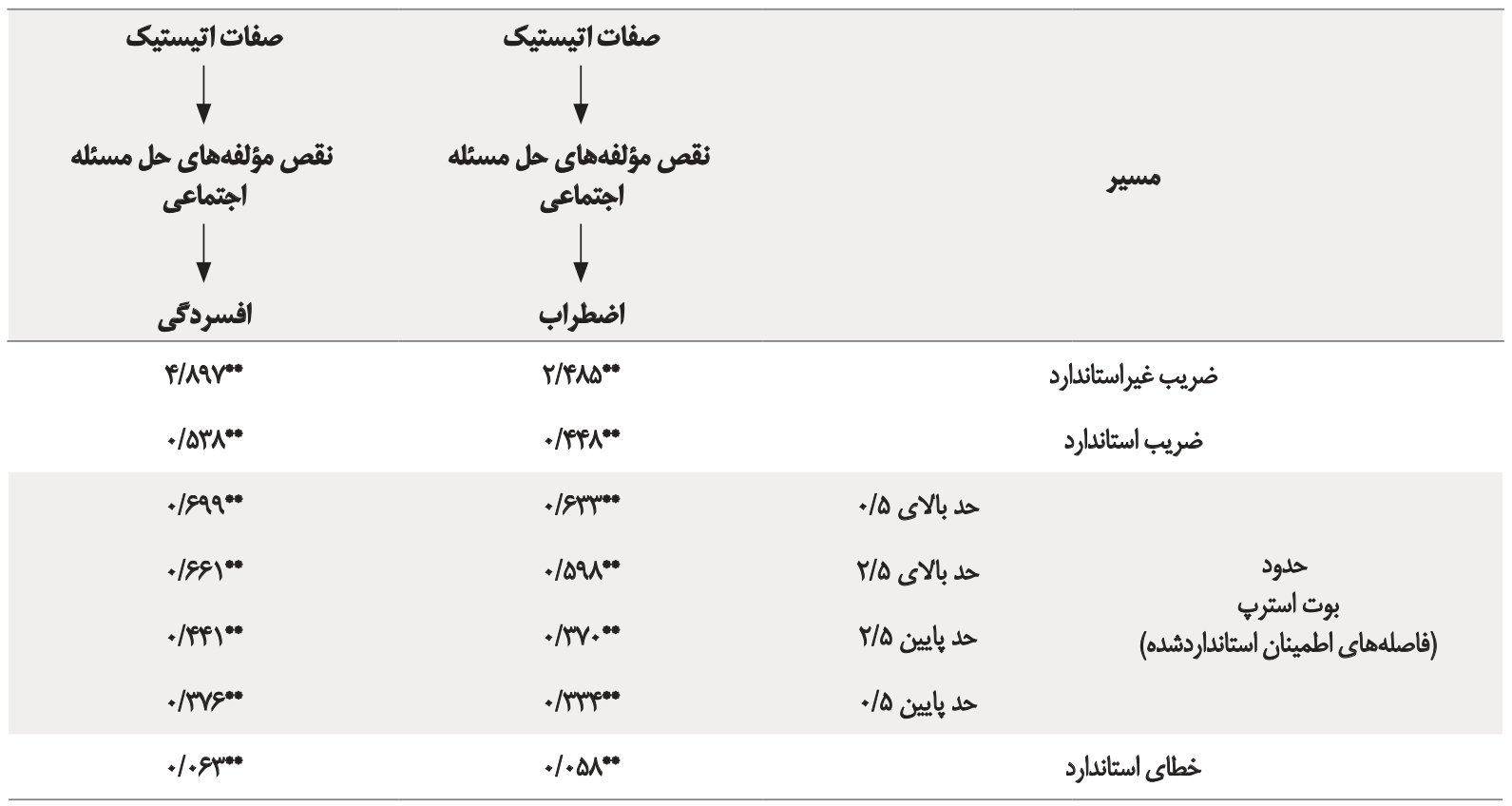

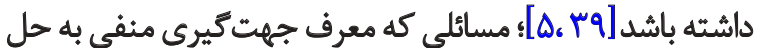

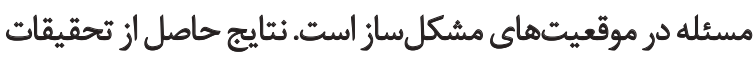

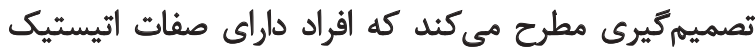

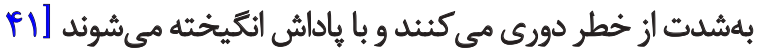

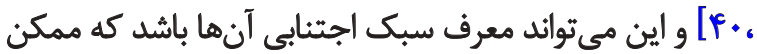

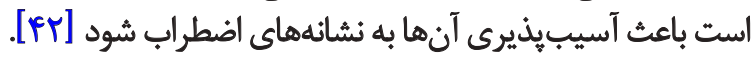

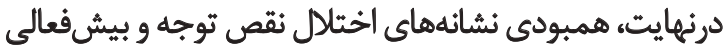

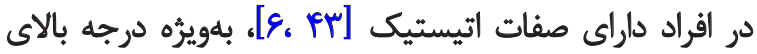

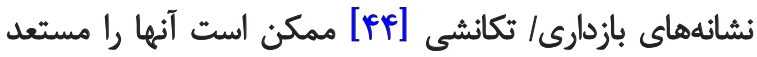
استفاده از سبك تكانشى/بي احتياطي كند.

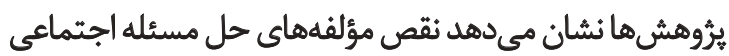

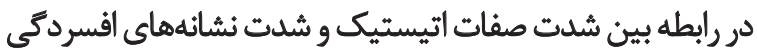

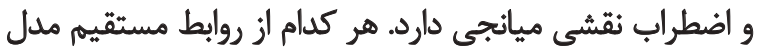

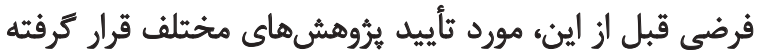

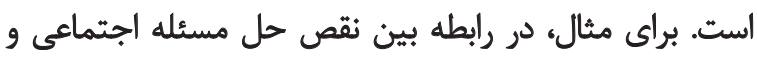

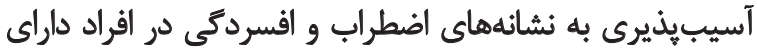

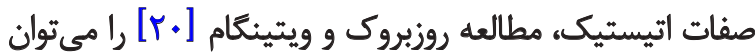

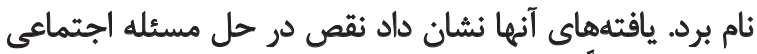

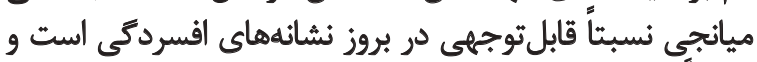

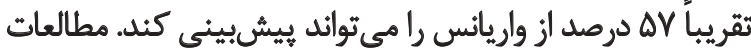

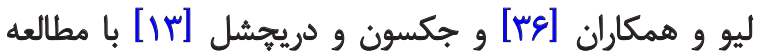

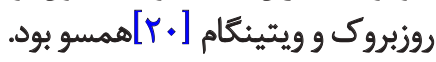

مطالعه حاضر هم تواند نوعى مطالعه ابتدايى و مهم در بررسى روني
تكانشى بـىاحتياط) را به كار مى تيرند و يا در سبكهاى حل

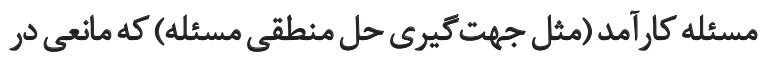

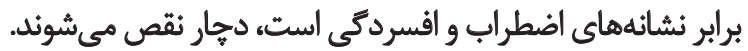

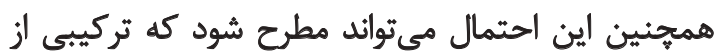

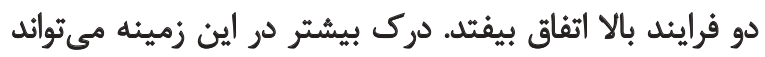

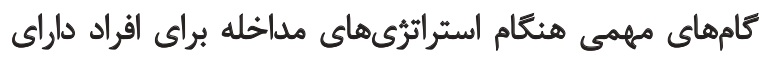

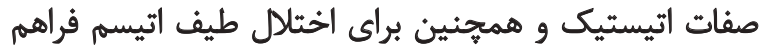

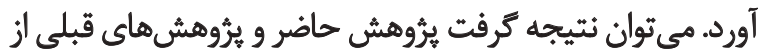

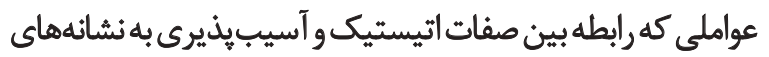

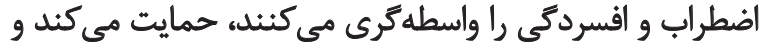

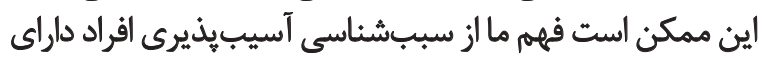

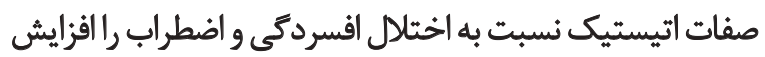

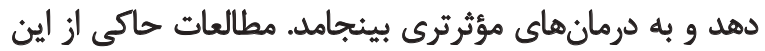

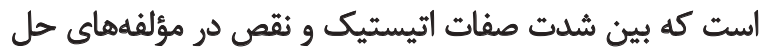

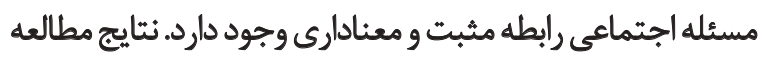

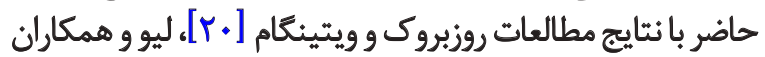

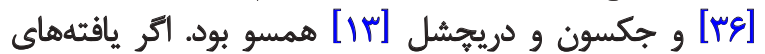

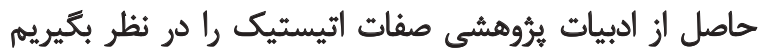

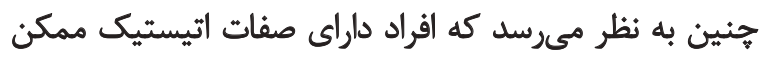

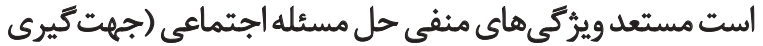

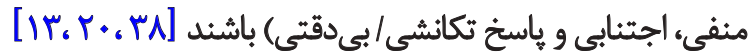

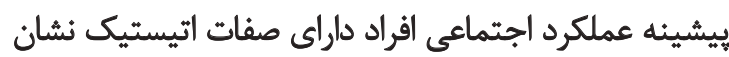

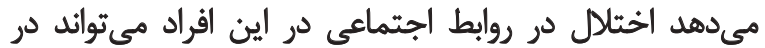
برداشت از بى كفايتى اجتماعى و خودكارآمدى ضعيف ريش إيشه 
نقش واسطهاي نقص مؤلفههاى حل مسئله اجتماعى در ارتباط

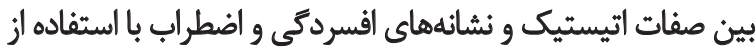

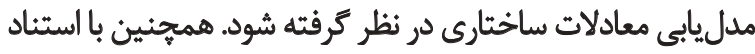

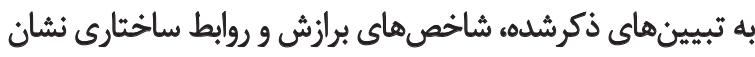

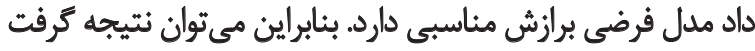

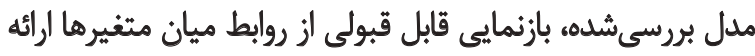

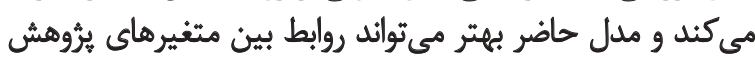

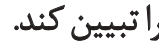
تثيجليرى

مطالعه حاضر محدوديتهايي داشت. با توجه به اينكه هما مانه

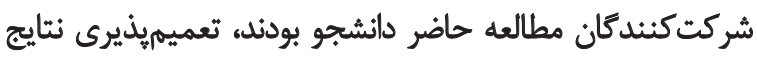

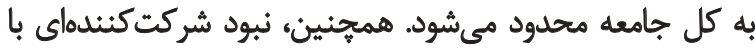

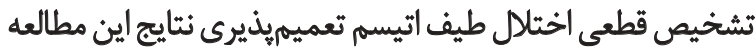

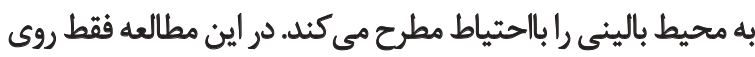

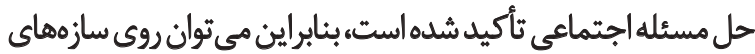

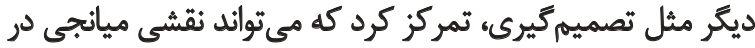

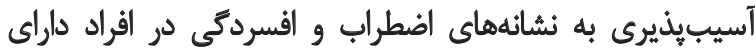

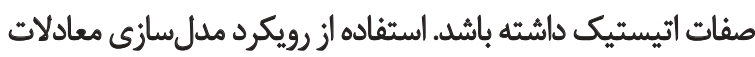

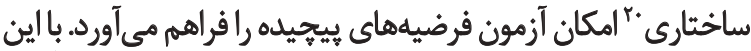

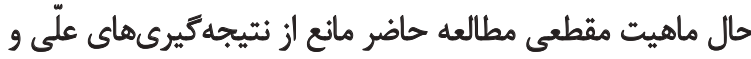

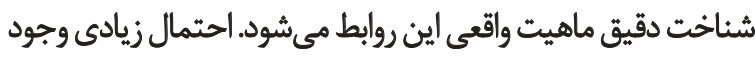

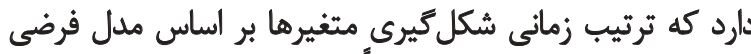

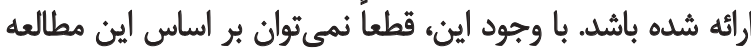
حالتهاى جايكزّين ديكًر را رد كرد.

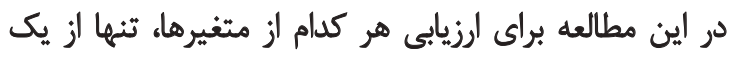

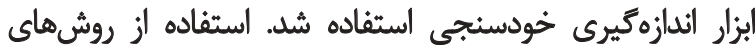

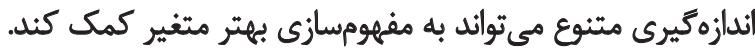

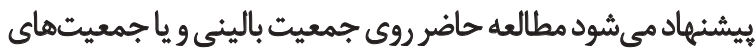

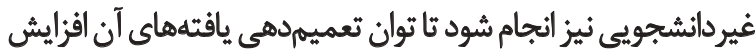

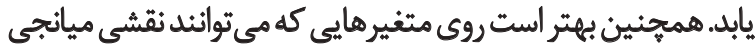

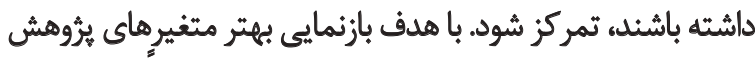

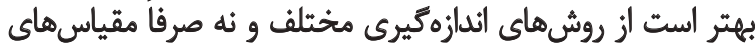
خودسنجى استفاده كرد.

بدينوسيله از شركت كنيدكان اين برؤوهش كه ما را يارى رساندند

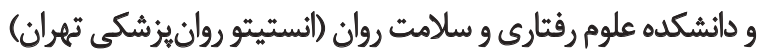

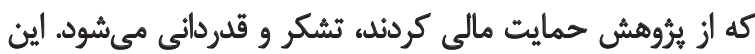

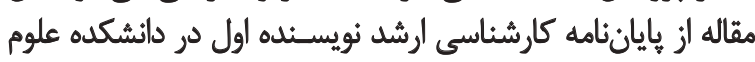

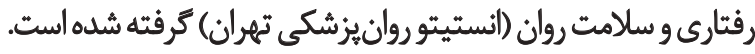
بنابه اظهار نويسنده مسئول مقاله، تعارض منافع وجود نداشته است 


\section{References}

[1] American Psychiatric Association. Diagnostic and statistical manual of mental disorders, (DSM-5®). New York: American Psychiatric Pub; 2013.

[2] Constantino JN, Todd RD. Autistic traits in the general population. Archives of General Psychiatry. 2003; 60(5):524. doi: 10.1001/ archpsyc.60.5.524

[3] Ronald A, Happe F, Price TS, Baron Cohen S, Plomin R. Phenotypic and genetic overlap between autistic traits at the extremes of the general population. Journal of the American Academy of Child \& Adolescent Psychiatry. 2006; 45(10):1206-14. doi: 10.1097/01.chi.0000230165.54117.41

[4] Robinson EB. Evidence that autistic traits show the same etiology in the general population and at the quantitative extremes $(5 \%$, 2.5\%, and 1\%). Archives of General Psychiatry. 2011; 68(11):1113. doi: 10.1001/archgenpsychiatry.2011.119

[5] Sterling L, Dawson G, Estes A, Greenson J. Characteristics associated with presence of depressive symptoms in adults with autism spectrum disorder. Journal of Autism and Developmental Disorders. 2007; 38(6):1011-8. doi: 10.1007/s10803-007-0477-y

[6] Leyfer OT, Folstein SE, Bacalman S, Davis NO, Dinh E, Morgan J, et al. Comorbid psychiatric disorders in children with autism: Interview development and rates of disorders. Journal of Autism and Developmental Disorders. 2006; 36(7):849-61. doi: 10.1007/ s10803-006-0123-0

[7] Lugnegård T, Hallerbäck MU, Gillberg C. Psychiatric comorbidity in young adults with a clinical diagnosis of Asperger syndrome. Research in Developmental Disabilities. 2011; 32(5):19107. doi: $10.1016 /$ j.ridd.2011.03.025

[8] Ghaziuddin M. Mental health aspects of autism and asperger syndrome. London: Jessica Kingsley Publishers; 2005.

[9] Kunihira Y, Senju A, Dairoku H, Wakabayashi A, Hasegawa T. Autistic traits in non autistic Japanese populations: Relationships with personality traits and cognitive ability. Journal of Autism and Developmental Disorders. 2006; 36(4):553-66. doi: 10.1007/ s10803-006-0094-1

[10] Gillberg C, Billstedt E. Autism and asperger syndrome: Coexistence with other clinical disorders. Acta Psychiatrica Scandinavica. 2000; 102(5):321-30. doi: 10.1034/j.1600-0447.2000.102005321.x

[11] Matson JL, Nebel Schwalm MS. Comorbid psychopathology with autism spectrum disorder in children: An overview. Research in Developmental Disabilities. 2007; 28(4):341-52. doi: 10.1016/j.ridd.2005.12.004

[12] Ghaziuddin M, Ghaziuddin N, Greden J. Depression in persons with autism: Implications for research and clinical care. Journal of Autism and Developmental Disorders. 2002; 32(4):299-306. Doi: $10.1023 / a: 1016330802348$

[13] Jackson SLJ, Dritschel B. Modeling the impact of social problem-solving deficits on depressive vulnerability in the broader autism phenotype. Research in Autism Spectrum Disorders. 2016; 21:128-38. Doi: 10.1016/j.rasd.2015.10.002

[14] Nezu AM, Ronan GF. Social problem solving as a moderator of stress related depressive symptoms: A prospective analysis. Journal of Counseling Psychology. 1988; 35(2):134-8. doi: 10.1037/0022-0167.35.2.134
[15] Marx EM, Williams JM, Claridge GC. Depression and social problem solving. Journal of Abnormal Psychology. 1992; 101(1):78-86. doi: 10.1037/0021-843x.101.1.78

[16] Anderson RJ, Goddard L, Powell JH. Social problem solving processes and mood in college students: An examination of self report and performance based approaches. Cognitive Therapy and Research. 2007; 33(2):175-86. doi: 10.1007/s10608-007-9169-3

[17] Becker Weidman EG, Jacobs RH, Reinecke MA, Silva SG, March JS. Social problem solving among adolescents treated for depression. Behaviour Research and Therapy. 2010; 48(1):11-8. doi: 10.1016/j.brat.2009.08.006

[18] D'Zurilla TJ, Chang EC, Nottingham EJ, Faccini L. Social problem solving deficits and hopelessness, depression, and suicidal risk in college students and psychiatric inpatients. Journal of Clinical Psychology. 1998; 54(8):1091-107. doi: 10.1002/(sici)10974679(199812)54:8<1091::aid-jclp9>3.0.co; 2-j

[19] Argus G, Thompson M. Perceived social problem solving, perfectionism, and mindful awareness in clinical depression: An exploratory study. Cognitive Therapy and Research. 2007; 32(6):745-57. doi: 10.1007/s10608-006-9102-1

[20] Rosbrook A, Whittingham K. Autistic traits in the general population: What mediates the link with depressive and anxious symptomatology. Research in Autism Spectrum Disorders. 2010; 4(3):415-24. doi: 10.1016/j.rasd.2009.10.012

[21] Baron Cohen S. The autism-spectrum quotient (AQ): Evidence from asperger syndrome/high functioning autism, males and females, scientists and mathematicians. Journal of Autism and Developmental Disorders. 2001; 31(1):5-17. PMID: 11439754

[22] Nejatisafa A, Kazemibejestani SMR, Alageband J. [Autistic traits in adults: Evidence for autism continuum hypothesis (Persian)]. Advances in Cognitive Science. 2001; 5(3):34-9.

[23] D'Zurilla TJ, Nezu AM, Maydeu Olivares A. Social problemsolving inventory revised: Technical manual. North Tonawanda: Multi Health Systems; 2002

[24] Mokhberi A, Dortaj F, Darehkordi A. [Questionnaire, psychometrics, and standardization of indicators of social problem solving ability (Persian)]. Training Measurement. 2011; 1(4):55-72.

[25] Beck AT, Steer RA. Manual for the revised Beck depression inventory. San Antonio: Psychological Corporation; 1987.

[26] Stefan Dabson K, Mohammadkhani P, Massah Choulabi O. [Psychometrics characteristic of Beck Depression Inventory-II in patients with magor depressive disorder (Persian)]. Archives of Rehabilitation. 2007; 8:82.

[27] Beck AT, Epstein N, Brown G, Steer RA. An inventory for measuring clinical anxiety: Psychometric properties. Journal of Consulting and Clinical Psychology. 1988; 56(6):893-7. doi: $10.1037 / 0022-006 x .56 .6 .893$

[28] Kaviani H, Mousavi AS. [Psychometric properties of the Persian version of Beck Anxiety Inventory (BAI) (Persian)]. Tehran University Medical Journal TUMS Publications. 2008; 66(2):13640

[29] Tabachnick BG, Fidell LS, Osterlind SJ. Using multivariate statistics. New York: HarperCollins College Publishers: 1996.

[30] Kline RB. Principles and practice of structural equation modeling. New York: Guilford publications; 2015. 
[31] Chou CP, Bentler PM. Estimates and tests in structural equation modeling. In: Hoyle RH, editor. Structural equation modeling: Concepts, issues, and applications. Thousand Oaks: Sage Publications; 1995.

[32] Hu L, Bentler PM. Fit indices in covariance structure modeling: Sensitivity to underparameterized model misspecification. Psychological Methods. 1998; 3(4):424-53. doi: 10.1037/1082989x.3.4.424

[33] Preacher KJ, Hayes AF. Asymptotic and resampling strategies for assessing and comparing indirect effects in multiple mediator models. Behavior Research Methods. 2008; 40(3):879-91. doi: 10.3758/brm.40.3.879

[34] Kevin EK. Using mplus for structural equation modeling: A researcher's guide. Thousand Oaks: Sage Publications; 2015. doi: $10.4135 / 9781483381664$

[35] Zarekar A, Demehri F, RostamNia S, Rasouli AA. [Examining the relationship of autistic spectrum traits and depression between the girl students of Ilam Medical Sciences university (Persian)]. Journal of Ilam University of Medical Sciences. 2014; 21(7):178-85

[36] Liew SM, Thevaraja N, Hong RY, Magiati I. The relationship between autistic traits and social anxiety, worry, obsessive compulsive, and depressive symptoms: Specific and non specific mediators in a student sample. Journal of Autism and Developmental Disorders. 2014; 45(3):858-72. doi: 10.1007/s10803-014-2238-Z

[37] D'Zurilla TJ, Nezu AM. Development and preliminary evaluation of the Social Problem Solving Inventory. Psychological Assessment. 1990; 2(2):156-63. doi: 10.1037/1040-3590.2.2.156

[38] Goddard L, Howlin P, Dritschel B, Patel T. Autobiographical memory and social problem solving in asperger syndrome. Journal of Autism and Developmental Disorders. 2006; 37(2):291-300. doi: $10.1007 /$ s10803-006-0168-0

[39] Vickerstaff S, Heriot S, Wong M, Lopes A, Dossetor D. Intellectual ability, self perceived social competence, and depressive symptomatology in children with high functioning autistic spectrum disorders. Journal of Autism and Developmental Disorders. 2006; 37(9):1647-64. doi: 10.1007/s10803-006-0292-x

[40] Luke L, Clare ICH, Ring H, Redley M, Watson P. Decision-making difficulties experienced by adults with autism spectrum conditions. Autism. 2011; 16(6):612-21. doi: 10.1177/1362361311415876

[41] South M, Chamberlain PD, Wigham S, Newton T, Le Couteur $\mathrm{A}, \mathrm{McC}$ nachie $\mathrm{H}$, et al. Enhanced decision making and risk avoidance in high functioning autism spectrum disorder. Neuropsychology. 2014; 28(2):222-8. doi: 10.1037/neu0000016

[42] Mayes SD, Calhoun SL, Murray MJ, Zahid J. Variables associated with anxiety and depression in children with autism. Journal of Developmental and Physical Disabilities. 2011; 23(4):325-37. doi: 10.1007/s10882-011-9231-7

[43] Sinzig J, Morsch D, Bruning N, Schmidt MH, Lehmkuhl G. Inhibition, flexibility, working memory and planning in autism spectrum disorders with and without comorbid ADHD symptoms. Child and Adolescent Psychiatry and Mental Health. 2008; 2(1):4. doi: 10.1186/1753-2000-2-4

[44] Ames CS, White SJ. Brief report: Are ADHD traits dissociable from the autistic profile? Links between cognition and behaviour. Journal of Autism and Developmental Disorders. 2010; 41(3):35763. doi: 10.1007/s10803-010-1049-0 
